POS PROCEEDINGS

\title{
Keeping the background low: production and testing of the GERDA Phase II detectors
}

Sabine Hemmer for the GERDA collaboration*

Univ. di Padova - INFN-Padova

E-mail: sabine.hemmer@pd.infn.it

The GERmanium Detector Array (GERDA) experiment at the INFN Laboratori Nazionali del Gran Sasso searches for neutrinoless double beta decay of ${ }^{76} \mathrm{Ge}$. In a future phase of the experiment, $\sim 20 \mathrm{~kg}$ of newly produced Broad Energy Germanium (BEGe) detectors will be deployed. A summary of the detector production process is given and first results of the detector acceptance testing are presented.

XV International Workshop on Neutrino Telescopes

March 11-15 2013

Venice, Italy

${ }^{*}$ Speaker. 


\section{Neutrinoless Double Beta Decay}

Neutrino-accompanied double beta decay $(2 v \beta \beta)$ has been observed for several nuclei [1] that cannot decay via single beta decay. In this standard-model allowed decay, the nucleus undergoes $2 v \beta \beta$ under emission of two electrons and two antineutrinos $\bar{v}_{e}$. In the case of neutrinoless double beta decay $(0 v \beta \beta)$, on the other hand, the nucleus decays under emission of two electrons without emission of $\bar{v}_{e}$. This process violates the lepton number by two units and is not allowed by the Standard Model. Current limits for the half-life of $0 v \beta \beta$ are of the order $10^{24}-10^{25} \mathrm{yr}[2,3,4,5$, 6]. A claim for a discovery of $0 v \beta \beta$ of ${ }^{76} \mathrm{Ge}$ was made by a subgroup of the Heidelberg-Moscow experiment $[7,8]$.

\section{The GERDA Experiment}

GERDA $[9,10]$ is an experiment designed for the search for $0 v \beta \beta$ of ${ }^{76} \mathrm{Ge}$. It is located in the INFN Gran Sasso underground laboratories. In its present configuration, the sensitivity of the GERDA experiment is limited by the background.

The environmental background component is reduced by a graded shielding. The germanium detectors are submerged directly in a cryostat filled with liquid argon (LAr). The cryostat is surrounded by a tank containing ultra-pure water and equipped with photomultiplier tubes to detect the Čerenkov light of remaining cosmic muons. Material close to the detectors is minimized. All materials used to build the experiment were screened to guarantee their radio-purity.

Several techniques have been developed to reject remaining background events. In general, background events have a different topology than $0 v \beta \beta$ events. Most $0 v \beta \beta$ events will deposit their energy locally within a sphere with a radius of $\leq 1 \mathrm{~mm}$ due to the limited range of electrons in germanium. These are so-called single-site events, SSE. The main background contribution comes from Compton-scattered photons. Their energy deposits are usually separated by centimeters, producing so-called multi-site events, MSE. It is therefore very unlikely that more than one detector in the array has an energy deposit in the case of a signal event. So, an anti-coincidence cut between different detectors allows for further reduction of the background.

Background events can additionally be reduced using pulse shape analysis, PSA [11, 12]. In this case, the pulses generated by the detectors in response to the energy depositions are analyzed. This allows to distinguish between different event topologies and thus to discard background events.

A first phase of the GERDA experiment uses conventional coaxial germanium detectors [10]. For a second phase, a novel detector design was chosen: Broad Energy Germanium, BEGe, detectors. They allow an improved background index, $B I$, and energy resolution.

A schematic drawing of a BEGe detector is depicted in Fig. 1a. The $\mathrm{n}^{+}$surface covers almost the complete surface of these $\mathrm{p}$-type detectors, whereas the $\mathrm{p}^{+}$contact is very small. This leads to a peculiar shape of the electric field, which allows a very effective PSA and very good distinction between SSE and MSE. Details can be found elsewhere [13, 14, 15, 16].

The excellent PSA performance together with an instrumentation of the LAr to read out the scintillation light will allow to reach a $B I$ as low as $10^{-3} \mathrm{cts} /(\mathrm{keV} \cdot \mathrm{kg} \cdot \mathrm{yr})$ in GERDA Phase II. With 




(a)

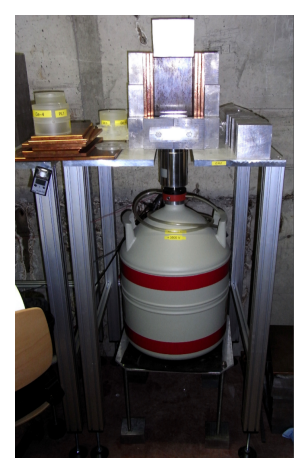

(b)

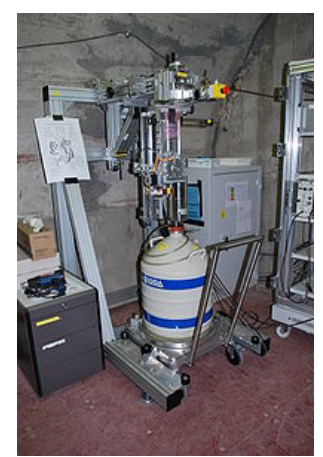

(c)

Figure 1: (a) A schematic drawing of a BEGe detector. (b) The fixed-source setup. (c) The scanning setup.

an exposure of $\sim 100 \mathrm{~kg} \cdot \mathrm{yr}$ this will allow to reach limits for the half-life of $0 v \beta \beta$ of ${ }^{76} \mathrm{Ge}$ of the order of $1.35 \cdot 10^{26} \mathrm{yr}(90 \%$ C.L.).

\section{Production and Testing of the GERDA Phase II Detectors}

Fast neutrons from the cosmic radiation produce radio-isotopes such as ${ }^{60} \mathrm{Co}$ and ${ }^{68} \mathrm{Ge}$ via spallation reactions in germanium. Their decay mimics signal events, increasing the $B I$. To guarantee the extremely low background level needed for the GERDA experiment, the production of the new BEGe detectors was carefully planned and carried out, reducing the exposure to cosmic radiation to a minimum. The material and later the diodes were always transported in a shielded container. At all production sites, underground storages were identified and the germanium was transported between the storage location and the production site on a daily basis. The exposure was rigorously tracked in order to estimate and control the activation. From originally $37.5 \mathrm{~kg}$ of $\mathrm{GeO}_{2}$ enriched in ${ }^{76} \mathrm{Ge}$ to a level of $\sim 88 \%, 30$ diodes with a total mass of $\sim 20 \mathrm{~kg}$ were produced. The cosmic activation was limited to $\sim 20-50{ }^{68} \mathrm{Ge}$ nuclei $/ \mathrm{kg}$ and $\sim 20-40{ }^{60} \mathrm{Co}$ nuclei/kg, resulting in an approximate $B I$ of $\sim 4 \cdot 10^{-4} \mathrm{cts} /(\mathrm{keV} \cdot \mathrm{kg} \cdot \mathrm{yr})$ and $\sim 4 \cdot 10^{-5} \mathrm{cts} /(\mathrm{keV} \cdot \mathrm{kg} \cdot \mathrm{yr})$, respectively.

The newly produced BEGe detectors have to meet very critical specifications, not only in terms of radio-purity and enrichment but also in terms of performance. A very good energy resolution as well as PSA performance is crucial to reach the required Phase II sensitivity. Detector parameters like active volume fraction, depletion voltage, and dead layer uniformity determine the detection efficiency for $0 v \beta \beta$ and have to be known very precisely. Therefore, an extensive acceptance measurement campaign was carried out at the HADES underground laboratory situated on the SCK.CEN premises in Mol, Belgium. Specifically developed setups allowed the complete characterization of all 30 detectors in a very tight time schedule, performing measurements on two or more detectors in parallel. The infrastructure is described in detail in [17]. It comprised two fixed-source measurement tables, used to expose the diode to a collimated or uncollimated calibration source in a few fixed positions, and three setups with a motor-controlled movable arm, designed to perform top and lateral surface scans of the diodes with a collimated $5 \mathrm{MBq}{ }^{241} \mathrm{Am}$ 




(a)

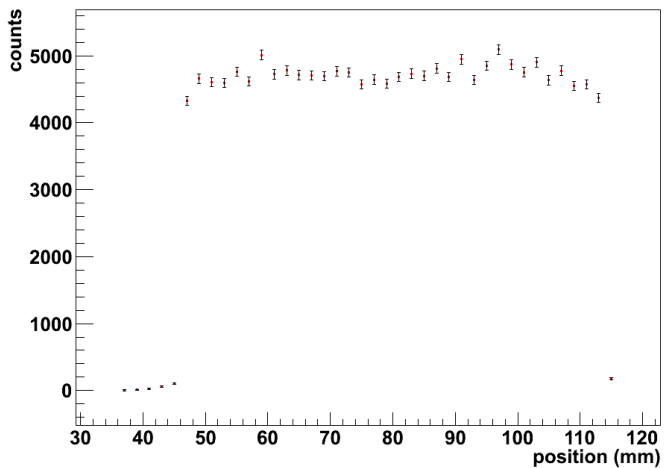

(b)

Figure 2: (a) The energy resolution in uncalibrated MCA counts as a function of the high voltage. A plateau is reached at full depletion at $\sim 2750 \mathrm{~V}$. (b) The peak rate at the $59 \mathrm{keV}$ photon line from ${ }^{241} \mathrm{Am}$ as a function of position for the top surface of a detector.

source and a precision of $1 \mathrm{~mm} / 1^{\circ}$. Examples of both setups can be seen in Fig. 1b and Fig. 1c, respectively.

For the testing procedure, a protocol developed with BEGe detectors produced from the residual material of the enrichment process [18] was followed.

The energy resolution was determined from a measurement with a ${ }^{60} \mathrm{Co}$ source at the operational voltage suggested by the manufacturer on the fixed-source measurement table. All 30 detectors showed an excellent energy resolution between $1.64 \mathrm{keV}$ and $1.86 \mathrm{keV}$, the average being $1.74 \mathrm{keV}$, at $1.33 \mathrm{MeV}$.

To determine the full depletion voltage, a high voltage scan was performed using a ${ }^{60}$ Co source with the fixed-source setup. The resolution, peak integral, and peak position of the $1173 \mathrm{keV}$ and $1333 \mathrm{keV}$ photon peaks in the uncalibrated energy spectrum in dependency of the high voltage were studied to determine the full depletion voltage. An example is shown in Fig. 2 a.

The active volume was extracted comparing measurements to Monte Carlo simulations in which the $\mathrm{n}^{+}$dead layer thickness was varied. For measurements with ${ }^{60} \mathrm{Co}$, the detection efficiency at the $1173 \mathrm{keV}$ and $1333 \mathrm{keV}$ photon peaks was directly compared to the Monte Carlo, while for the case of ${ }^{241} \mathrm{Am}$ and ${ }^{133} \mathrm{Ba}$, the active volume was deduced by comparing the relative intensity of two low energy photon peaks. The combination of the different approaches results in active volume fractions between $86 \%$ and $92 \%$ with a total uncertainty of the order of $3 \%$ in a first analysis of a subset of seven detectors.

The PSA performance was tested with a ${ }^{228}$ Th source. The survival fraction of events at the 2.6 MeV line is $13 \pm 5 \%$ when keeping $90 \%$ of events at the double escape peak at $1.6 \mathrm{MeV}$ in a first analysis of a subgroup of seven detectors.

Finally, a full set of surface scans was performed on the scanning tables to determine the detector dimensions and to test the charge collection and pulse shape parameter homogeneity. An example of a surface scan is depicted in Fig. $2 b$. 


\section{References}

[1] A.S. Barabash, Phys. Rev. C 81, 035501 (2010)

[2] H.V. Klapdor-Kleingrothaus et al., Eur. Phys. J. A 12, 147-154 (2001)

[3] C.E. Aalseth et al., Phys. Rev. D 65, 092097 (2002)

[4] A. Gando et al., Phys. Rev. C 85, 045504 (2012)

[5] M. Auger et al., Phys. Rev. Lett. 109, 032505 (2012)

[6] C. Arnaboldi et al., Phys. Rev. C 78, 035502 (2008)

[7] H.V. Klapdor-Kleingrothaus et al., Phys. Lett. B 586, 198-212 (2004)

[8] H.V. Klapdor-Kleingrothaus and I. V. Krivosheina, Mod. Phys. Lett. A 21, 1547-1566 (2006)

[9] I. Abt et al., preprint arXiv:hep-ex/0404039 (2004)

[10] K.H. Ackermann et al., Eur. Phys. J. C 73, 2330 (2013)

[11] B. Majorovits and H.V. Klapdor-Kleingrothaus, Eur. Phys. J. A 6, 463-469 (1999)

[12] D. González et al., Nucl. Instrum. Methods A 515, 634-643, (2003)

[13] D. Budjáš et al., JINST 4, P10007 (2009)

[14] M. Barnabé Heider et al., JINST 5, P10007 (2010)

[15] M. Agostini et al., JINST 6, P03005 (2011)

[16] M. Agostini et al., JINST 6, P04005 (2011)

[17] E. Andreotti et al., JINST 8, P06012 (2013)

[18] D. Budjáš et al., JINST 8, P04018 (2013) 